\title{
A Novel Approach for Examination of Visually Challenged Candidates by E-Evaluation Techniques
}

\author{
M. Dhanalakshmi \\ Associate Professor, Department of IT, JNTUHCEJ, INDIA
}

\begin{abstract}
The evaluation of physically challenged is always a challenging task as any evaluation of them is compared with that of normal candidates. The case of visually challenged is still more difficult as vision is a nunerouno sensor in the field of study and knowledge enhancement and evaluation of them on par with other candidate is very difficult. The aim of this paper is to present an approach for E-evaluation model for the visually challenged students/candidates for the screening tests conducted by the different examination authorities. The major attempt is made to use the personal computer and avoid the use of a scriber by the candidate so that candidate can take the exam independently. A portion of the PC keyboard is slightly modified in its software functionality to help them in undergoing the test. Also described is the functioning of this model of E-Evaluation and its relative advantages.
\end{abstract}

Keywords: E-evaluation, PC keyboard, visually challenged candidate

\section{INTRODUCTION}

Electronic evaluation (or e- evaluation) is a type of Technology supported evaluation where the medium of instruction is through computer and communication technologies. E-evaluation is a part of e-learning and evaluation is a process to assess a candidate. E-learning is benefiting the sections of the human society hitherto that was very difficult to make learning in those sections like people from rural areas, Working personal, Physically challenged etc... One of those vulnerable sections of the society is Visually Challenged Candidates (VCC). This paper gives a novel approach for the E-evaluation of the visually challenged people which can be compared to be on par with the examination of other normal candidates.

1.1 THE CHALLENGE OF A VCC :In one of the developing country like India, as per the 2001 population census [1], 10.635 million people (about 1\%) are visually impaired, i.e., one person in every hundred is suffering from visual impairment. It is an inhumane from our part if such a large chunk of population is denied the chances and rights on par with others. Most of these persons are not opting for higher education and thus for good jobs because of the obstacles in learning, qualifying and also related problems in writing exams. Some Universities do not admit the visually impaired at all, while some are giving them admissions with a limited option of subjects. Most of the reasons as told by them are the problems involved in evaluation \& teaching then on par with normal students.

Under this challenging environment, even when some VCCs get qualified in the University exams, next problem they face is the hurdle of screening tests or competitive exams conducted for the jobs by different recruiting agencies like Union and State Public service commissions, Companies, Boards etc. In one of the ad [2], a job recruiting agency prescribes the rules for VCCs as: 1) the candidate has to arrange candidate's own scribe/writer at candidate's own cost. 2) The scribe/writer must be in at least one grade junior in academic qualification to the candidate. 3) Scribe/writer should be one possessing marks of $50 \%$ or less. 4) Candidate should be from an academic discipline other than that of the candidate. 5) Both candidate and scriber shall have to give a suitable undertaking conforming that the scribe fulfils all the stipulated eligibility criteria. 6) They will be given 20 minutes extra for every one hour of the exams [3]. The careful observation of these above rules show us the difficulties faced by a VCC candidate.

In this technological age where a number of electronic gadgets are available and software has penetrated in to every aspect of our life, the methods of E-learning and E-evaluation can be used to reduce the hurdles of the VCC candidate [4][5]. This paper gives a model where in it explains an approach on how a VCC can take a screening test using E-gadgets and without any scriber's help.

\section{THE COMPONENTS OF THE E-EVALUATION MODEL}

In this model of E-evaluation for VCCs, the gadgets required are a personal computer with a keyboard, an earphone and a $\mathrm{CD} /$ storage devise (with E-evaluation software and question paper). The candidate undergoing exam has to use these gadgets during candidate's evaluation. This model is aimed for the objective type exams that are most common for the screening tests. The algorithm that is to be followed by the evaluation 
program software is presented in this paper and it explains how a VCC takes an exam and how candidate's evaluation is carried out without the help of any other person.

The components of this model are :- a) a computer with program software and question paper and answer sheet(QA sheet) in the CD, b) Earphones, c) Keyboard and d) Visually Challenged candidate taking the exam. The course of evaluation of VCC requires the complimentary use of these components as shown in Fig-1.

Fig-1

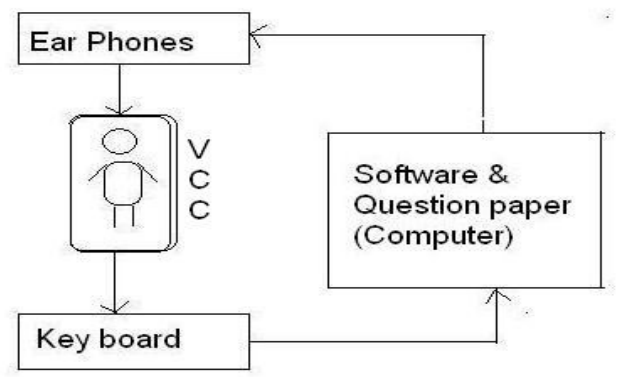

2.1 Computer -This is the platform on which a VCC takes this exam/screening test. It can be a general configuration Personal computer with a CPU, monitor, keyboard, CD player with read/write facility, earphone facility etc. This type of computer setup is now a common hardware easily available in any examination centre. 2.2 Keyboard - This is a single way interface between the software and the candidate by which VCC answers and controls the question paper. Only a small portion (right side of the board) of the ordinary computer keyboard is to be used by the candidate. The keys of this portion of the keyboard (Fig-2A) are to be pasted with symbols as shown in the Fig-2B below. On enough practice even this may not be required by the candidate. The functions of the symbols/keys are explained in below table.

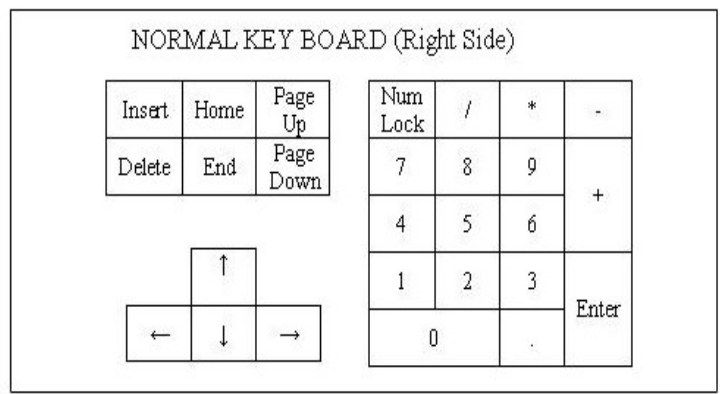

Fig-2A

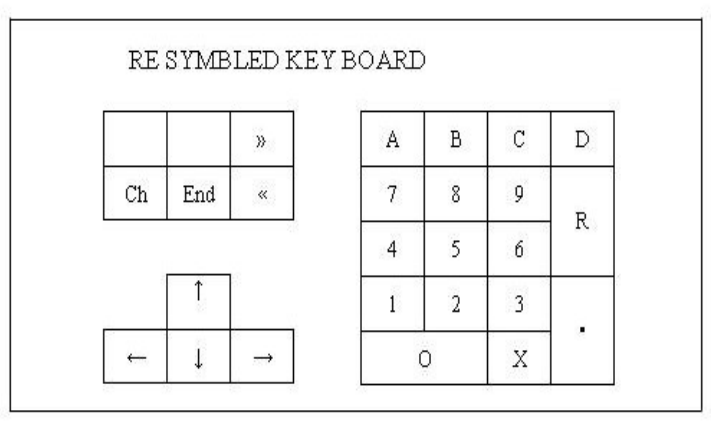

Fig-2B

The nornal keyboard with its general symbols is shown in Fig- 2A. The modified key board functions differently with its symbols changed as shown in Fig-2B. This is done by the program software of the CD when inserted into PC for the evaluation purpose. This modification is essential and critical as this is the media by which the VCC interacts with the QA sheet. The functions of the new symbols are shown in Fig-3.

Fig-3

\begin{tabular}{|c|l|}
\hline Symbol & \multicolumn{1}{|c|}{ Function } \\
\hline $\mathrm{A}, \mathrm{B}, \mathrm{C}, \mathrm{D}$ & Answers for the Question \\
\hline $0,1,2,3,4,5,6,7,8,9$ & Question No or other any No \\
\hline$\cdot$ & Enter to go next or whenever called \\
\hline$\uparrow, \downarrow, \rightarrow, \leftarrow$ & for previous or next Question \\
\hline ",$"$ & For previous or next section \\
\hline $\mathrm{R}$ & For repeating the question \\
\hline $\mathrm{X}$ & For canceling the answer \\
\hline $\mathrm{CH}$ & Check the already answered question \\
\hline $\mathrm{E}$ & End \\
\hline
\end{tabular}


2.3 Earphones-This is also a single way interface [6] between the software and the candidate by which VCC hears the instructions and questions from the $\mathrm{CD}$.

2.4 C.D./Storage device-This is the storage device and resembles in a way similar to a question paper and answer sheet to a normal candidate. This also contains the program software to run the exam and give instructions to the candidate. This is a password protected read-write device and two passwords are required to open it. One password has to be entered by the examiner / invigilator and the other by the VCC candidate. Candidates password can be given in candidate's hall ticket.

\section{Algorithm Of The Software}

The software developed for this e-evaluation uses the text reading software as a subroutine. Texts reading software is now-a-days became common and are used in many fields. The program software designed for our purpose reads the instructions and questions for the candidate. It takes the commands from the keyboard and operates the next function (i.e. marks/changes the answer sheet and reads the next question / message) as described in below algorithm. The answer sheet gets updated on the entering of keys-A, B, C or D. End of every question or instruction reading waits for a command from keyboard and every entry from keyboard results in reading of next question / instruction.

Proposed algorithm:

Step1: Enter the CD into Computer. I/ Auto playing CD and Software gets installed into the PC.

Step2: Enter the password of the Invigilator.

I/ Invigilator has to enter candidate's password and Time gets displayed and invigilator reads the same.

Step3: Enter the password of the VC candidate.

//Candidate has to enter candidate's password and now program opens

Step4: Enter the language code.

//For entering language code (optional), Eg: 1-English, 2-Hindi, 3-Telugu, 4-Tamil

Or this can be set with the invigilator from the application form)

Step5: Explains the details of the exam and question paper.

//Software explains the details of the exam, Question paper, Rules and Regulations etc...

I/All these are heard by candidates through ear phone.

Step6: Exam starts

//Reads first question

Step7: Candidate can do anyone of the following action

(i) Press $A$ or $B$ or $C$ or $D$ for answering the question or

(ii) $\quad$ Press $R$ for re-read the question or

(iii) Press $\longrightarrow \downarrow$ for go to next question or

(iv) Press $\uparrow$ for go to previous question or

(v) Press $>>$ for go to next section asks for $Q 1$ or

(vi) Press $<<$ for go to previous section $\longrightarrow$ Enter Q.No.

(vii) Press $X Y Z$ question number

After all questions are answered/read

(viii) Press $\mathrm{CH}$ to check the answered question

1. Reads Q.No. from start and Enter section $>>,<<$

2. After hearing the Question and Answer press. for "OK- No changes" or press * for "Cancel the Answer".

3. if $*$ is pressed, then enter the new answer - Press A or B or C or D

4. Press $\mathrm{CH}$ to go to next answered question - step (viii) repeats

5. Press . to go to next unanswered question.- step (ix) follows

(ix) Press . check the unanswered question

1. Reads unanswered questions from start

2. Step 7 repeats

Step8: Submit the answered paper or if time elapses the program will close

Step9: End 


\section{Exam Course}

Generally before the start of any exam, there will be some pre exam work. In most of the cases it involves: filling of hall ticket no, writing of name of exam, year, paper code etc. But in this case, the pre exam involves the entry of Passwords. The CD has to come with all the information filled in the exam application form of the VCC. As the CD is inserted in the computer, it auto plays the program. In this CD, the time duration of the exam is already set and the invigilator reads out the time to the candidate after entering his password. Then the candidate has to enter candidate's password. Before the actual start of exams, the instructions and the details of the question paper are to be read by the computer and the candidate hears it though the earphones.

With the pre exam work completed, the VCC is ready for the exam. At the exact time, exam starts with the reading of Question no-1. The candidate starts answering using the keyboard and the answer sheet in the CD gets updated as shown in the above algorithm. The exam ends automatically with the lapse of time as the time period is already set in the program. The Program automatically closes the CD with the end of the exam.

\subsection{EVALUATION OF THE EXAM PAPER}

The CD of the paper completed by VCC can be read /opened with a secured password for the evaluation purpose. There are two ways of evaluation of this paper:

1) Evaluation can be done by getting the QA sheet printed and then corrected in the normal procedure or

2) From this CD, a softcopy of the QA sheet has to be taken and giving this to the evaluation computer directly, we can get the result.

\subsection{ADVANTAGES OF THIS MODEL}

This model evaluation of a VCC has many advantages over other existing systems of evaluation. The advantages are:

1) It uses the existing computer hardware facilities at the examination center. Only ear phones and CDs are to be supplied by the Examination / recruiting agencies.

2) As there is no use of scribe, there is a total confidence on a candidate's performance and evaluation

3) As no external help is required for the VCC candidate, this model makes candidate independent like normal candidate. Also the burden of getting the proper scribe [3] is taken away from the candidate.

4) Candidate can practice this model if candidate is given a model $C D$ with the issue of hall ticket and candidate can learn it with least difficulty as number of gadgets to be operated is few.

5) Due to the use of limited no of keys and that to a particular part of the keyboard, this model is user friendly.

6) It is a language independent; an objective type test in any language can be conducted for the VCC, provided the text reading software is available in that language.

For getting better results this model is to be practiced by the candidate before exam. This can be made practiced in VCC rehabilitation centers, colleges, etc. to get accustomed by the candidate. A practice CD is to be issued by examination/recruiting agency with the issue of candidate's hall ticket. Whether extra time can be given to the VCC undergoing evaluation in this model can be decided by an expert committee involving VCC. An efficient text reading software is to be selected for a success of this model.

\section{Conclusion}

An effort has been made to evolve a friendly and secured model to evaluate a Visually Challenged Candidate. The approach of this model is created by making use of the generally available e-gadgets and the software technology to make a VCC to take an objective type test without the help of a scriber/writer. Further attempts are to be made to realize software and make a sample survey to tune the program for its effectiveness.

\section{References}

[1]. Census of India-2001.

[2]. Application details for the post of probationary officer in State bank of India.

[3]. News paper clippings:- Times of India-18may 2009, Expess News service 15 may 2009, IANS 8 march 2008.

[4]. Proceedings of International seminar on empowering Visually Impaired through ICT, April 15-16-2009.

[5]. Lorentz J ntschi, Sorana D. Bolboac, Monica M. Marta, and Alexandru Laszlo, 'E-Learning and E- Evaluation: A Case Study', HIS 2008, Krakow, Poland, May 25-27, 2008.

[6]. Daniel Fallman, Oskar Fjellström \& Kent Lindbergh, 'AudioIndex: Library Access for the Visually Challenged Using an RFIDbased Point and Listen Interface'. 